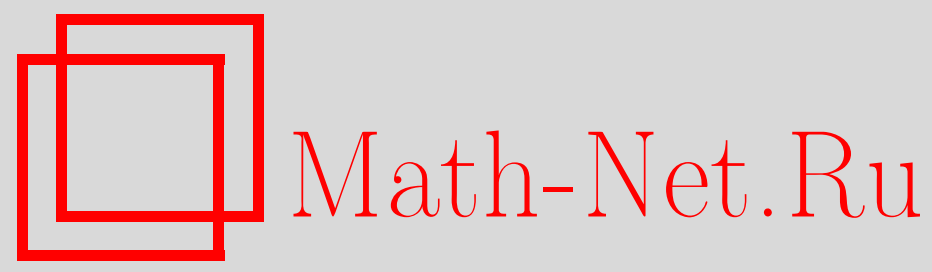

А. С. Меретин, Е. Б. Савенков, Моделирование термопороупругой среды с учетом разрушения, Матем. моделирование, 2020, том 32, номер 7, 59-76

DOI: https://doi.org/10.20948/mm-2020-07-04

Использование Общероссийского математического портала Math-Net.Ru подразумевает, что вы прочитали и согласны с пользовательским соглашением http://www.mathnet.ru/rus/agreement

Параметры загрузки:

IP : 54.80 .73 .141

26 апреля 2023 г., $07: 01: 55$ 


\title{
МОДЕЛИРОВАНИЕ \\ ТЕРМОПОРОУПРУГОЙ СРЕДЫ С УЧЕТОМ РАЗРУШЕНИЯ
}

(C) 2020 2. А.С. Меретин ${ }^{1}$, Е.Б. Савенков ${ }^{2}$

${ }^{1}$ Московский физико-технический институт

${ }^{2}$ ИПМ им. М.В. Келдыша РАН

meretin.as@cet-mipt.ru

e.savenkov@gmail.com

DOI: $10.20948 / \mathrm{mm}-2020-07-04$

Рассматриваются вопросы математического моделирования термопороупругой среды с учетом ее разрушения. Используемая модель обобщает классическую модель Био поведения пороупругой среды на случай учета термоупругих эффектов. Для описания разрушения среды используется подход континуальной механики разрушения, в рамках которого состояние среды описывается скалярным полем повреждаемости, от которого, в свою очередь, зависят упругие и фильтрационно-емкостные свойства среды. Система уравнений модели состоит из фундаментальных законов сохранения массы, импульса и энергии и замыкается термодинамически согласованными определяющими соотношениями. При этом выражение для энергии среды учитывает её изменение за счет образования зон разрушений. Вычислительный алгоритм основан на методе конечных элементов. Используется «монолитный» подход, который предполагает, что все группы уравнений (механика, теплоперенос, фильтрация) модели решаются одновременно без расщепления по физическим процессам и/или итераций между группами уравнений. Система уравнений термопороупругости аппроксимируется полностью неявной схемой. Эволюция параметра повреждаемости в зависимости от напряженно-деформированного состояния среды может описываться как в рамках мгновенной кинетики, так и в рамках кинетики с конечным временем. В работе кратко описана используемая математическая модель. Подробно описан вычислительный алгоритм и особенности его реализации. Значительная часть работы посвящена применению разработанных подходов для решения ряда задач как в модельных, так и в реалистичных трехмерных постановках. В качестве основной области применения построенной модели и алгоритма рассматривается анализ задач геомеханики, характерных для тепловых методов увеличения нефтеотдачи и требующих согласованного описания динамики упругих, фильтрационных и тепловых полей с учетом разрушения среды.

Ключевые слова: термопороупругость, модель Био, разрушение, термодинамическая согласованность, процедура Колмана-Нолла, метод конечных элементов.

\section{SIMULATION OF THERMOPOROELASTIC MEDIUM WITH DAMAGE}

\author{
A.C. Meretin ${ }^{1}$ E.B. Savenkov ${ }^{2}$ \\ ${ }^{1}$ Moscow Institute of Physics and Technology \\ ${ }^{2}$ Keldysh Institute of Applied Mathematics of RAS
}


In this paper we consider a numerical simulation of thermoporoelastic medium with damage. The model generalizes the classical Biot model for poroelastic medium evolution to the case of thermoelastic effects. Damage of the medium is simulated within the framework of continuum damage mechanics, where state of the medium is described by scalar damage parameter, which affects on elastic and flow properties. The system of governing equations consists of fundamental mass, momentum and energy conservation laws and is closed by thermodynamically-consistent constitutive relations. Moreover, the energy expression takes into account its changing due to formation of damaged zones. The computational algorithm is based on the finite element method. The "monolithic" approach is used, which assumes that all groups of equations (mechanics, heat transfer and flow) are solved simultaneously without splitting on physical processes and / or iteration coupling between groups of equations. The model is approximated by a fully implicit scheme. Damage parameter evolution depending on the stress-strain state can be described within the framework of instant or finite-time kinetics. The paper briefly describes the mathematical model. The computational algorithm and its implementation features are described in detail. Significant part of the work is related to the application of the developed approaches for solving a number of model and realistic threedimensional problems. The main field of the model and algorithm application is the analysis of geomechanical problems of thermal enchanced oil recovery methods, which require a consistent description of the elastic, filtration and thermal fields dynamics coupled with medium damage evolution.

Keywords: thermoporoelasticity, Biot, damage, thermodynamic consistency, ColemanNoll procedure, finite element method.

\section{1. Введение}

Настоящая работа посвящена вопросам математического моделирования динамики термопороупругой среды с учетом разрушения. Используемая математическая модель является обобщением классической модели М. Био пороупругой среды. Модель является термодинамически согласованной в смысле выполнения основных законов сохранения массы, энергии и импульса, а также энтропийного неравенства в нужном виде, см [1].

В рамках модели Био среда описывается как совокупность двух взаимопроникающих континуумов («фаз») - упругого «скелета» (твердой фазы) и насыщающей его жидкости (подвижной фазы). Под воздействием приложенных к ней внешних нагрузок динамика среды определяется совместной эволюцией напряженно-деформированного состояния пористого проницаемого деформируемого твердого тела и полей давления фильтрующегося в нем флюида. Уравнения модели представляют собой сильно связанную систему уравнений термомеханики и фильтрации. Типичными приложениями модели Био являются задачи гидрогеологии [2], анализ процессов в костных и мягких биологических тканях $[3,4]$, гидрогелях и многие другие. 
Следует отметить, что модель Био является не единственной известной моделью для описания динамики пороупругой среды. В частности, следует отметить модель, предложенную в работах В.Н. Доровского и его коллег. Модель является термодинамически согласованной и получена в рамках развиваемой авторами теории многоскоростного континуума [5-7]. Особенностью данной модели является то, что пороупругая среда описывается тремя упругими модулями, однозначно определяемыми по скоростям распространения упругих волн [5]. Данная модель применяется для описания движения волн Стоунли [6], в задачах сейсморазведки [7] и других областях.

Одним из важных приложений моделей рассматриваемого класса являются задачи нефтяной геомеханики, особенно применительно к анализу современных методов повышения нефтеотдачи, в частности, так называемых тепловых методов, суть которых заключается в подводе тепла в пласт путем закачки теплоносителя либо при помощи кондуктивного теплообмена [8]. При применении термических методов воздействия процесс разработки сопровождается проявлением таких эффектов, как изменение свойств пластового флюида, протекание химических реакций, изменение фильтрационноемкостных свойств пласта за счет изменения степени подвижности заполняющего поры флюида и образования зон микротрещиноватости. Расширение представлений о сложных взаимосвязанных процессах, сопровождающих тепловое воздействие на нефтеносный пласт, возможно только при использовании комплексного описания основных процессов, происходящих в пласте, включая геомеханические эффекты и разрушение пласта.

Существует ряд моделей, позволяющих учитывать указанные явления. В большинстве таких моделей повреждаемость среды описывается в рамках теории континуального разрушения $[9,10]$, когда в число термодинамических параметров, описывающих состояние среды, вводится так называемый параметр повреждаемости. Эволюция этого параметра определяется тем или иным кинетическим уравнением, которое связывает значение параметра (или скорость его изменения) с напряженно-деформированным состоянием среды. В свою очередь, этот же параметр входит в основные определяющие соотношения и влияет на фильтрационно-емкостные, термодинамические и геомеханические параметры нефтяного пласта.

В настоящей работе используется предложенная в [1] термодинамически согласованная математическая модель для описания эволюции термопороупругой среды с учетом континуального разрушения. Особенностью модели является то, что, с одной стороны, она получена в рамках строгого термодинамического формализма, гарантирующего ее корректность, а с другой - может быть применена на практике для решения задач в реалистичных постановках. 
В рассматриваемом приближении система уравнений пороупругости представляет собой связанную задачу для эллиптического уравнения (описывающего напряженно деформированное состояние насыщенной среды) и параболических уравнений (описывающих законы сохранения массы флюида и энергии системы). Для решения указанной системы уравнений могут применяться различные методы, среди которых отметим метод конечных объемов, метод конечных элементов и метод граничных интегральных уравнений.

Для аппроксимаций по времени чаще всего используются полностью неявные схемы, они же будут использованы и в данной работе. При этом полная система уравнений может аппроксимироваться как одно целое, либо задача «развязывается» и ее решение получается в ходе тех или иных итераций между группами уравнений фильтрации и упругости. Последний подход чаще всего применяется в случае, если для решения задачи используются две различные программы для расчета фильтрационной части (например, с помощью промышленного симулятора фильтрации) и для решения задачи теории упругости. Построение эффективных итерационных методов решения таких задач является отдельной проблемой, см., например, [11].

Характерным свойством системы уравнений пороупругости в рассматриваемом приближении является то, что после аппроксимации по времени система уравнений имеет вид задачи о седловой точке [12]. В этом случае для устойчивости решения задачи как в континуальном, так и в дискретном случае необходимо выполнение так называемых inf-sup-условий (условия Ладыженской-Бабушки-Бреззи) [12]. При нарушении этих условий в задачах пороупругости возникают численные неустойчивости и эффекты «блокировки» («locking») конечномерного решения, особенно в несжимаемом пределе (см. [13] и ссылки там). Простейшим примером конечного элемента, удовлетворяющего этим условиям является элемент Тэйлора-Худа [14], в котором для аппроксимации перемещений используются конечные элементы второго порядка, а для аппроксимации давления - первого. При этом обе системы функций заданы на одном и том же разбиении области на конечные элементы. Данный конечный элемент гарантирует оптимальную скорость сходимости, в том числе и в задачах пороупругости [15]. Этот подход будет использоваться и в данной работе.

Альтернативным подходом является такая регуляризация исходной задачи (см., напр., [16-18]), для которой inf-sup условия выполняются при использовании стандартных пар пространств (конечные элементы одинакового, первого, порядка и для поля перемещений, и для давления и температуры). 
В настоящей работе применяется метод конечных элементов. К его достоинствам можно отнести достаточно формальный способ построения конечномерной задачи и эффективность при использовании неструктурированных расчетных сеток. В отличии от ряда других работ (см., например, $[19,20])$, в настоящей работе задача рассматривается в полностью связанной («монолитной») постановке, когда все уравнения модели решаются одновременно, в рамках единой системы уравнений. Таким образом, не происходит итерационного связывания между группами уравнений теории упругости, фильтрации и баланса энергии, что особенно важно в связи с многодисциплинарностью задачи и учетом дополнительных эффектов (непосредственно разрушение и зависимость от него параметров модели).

Основная цель работы - описание модели, комплекса вычислительных алгоритмов и их программной реализации, которые дают представление об их возможностях и показывают применимость рассмотренных подходов для решения задач в постановках, приближенных к реальным.

\section{2. Математическая модель}

Рассмотрим термопороупругую среду, состоящую из двух континуумов - пористого деформируемого скелета и однофазного слабосжимаемого флюида. Система уравнений, описывающая поведение такой среды, имеет следующий вид [1]:

$$
\begin{aligned}
& \frac{\partial m_{f}}{\partial t}+\operatorname{div}\left(\rho_{f} \mathbf{w}\right)=0, \\
& \operatorname{div} \boldsymbol{\sigma}=0, \\
& \frac{\partial\left(m_{s} e_{s}+m_{f} e_{f}\right)}{\partial t}+\operatorname{div}\left(\rho_{f} e_{f} \mathbf{w}\right)=\operatorname{div}(-p \mathbf{w})-\operatorname{div}(\mathbf{q}),
\end{aligned}
$$

где $m_{\alpha}$ - масса фазы $\alpha$ в некотором элементарном объеме, $\rho_{f}-$ плотность флюида, $\mathbf{w}=-(\mathbf{k} / \mu) \operatorname{grad}(p)-$ скорость фильтрации, $\boldsymbol{\sigma}$ - тензор полных напряжений, $e_{\alpha}$ - удельная внутренняя энергия, $p$ - поровое давление, $\mathbf{q}=-\boldsymbol{\kappa} \operatorname{grad}(T)$ - вектор потока тепла, $\mathbf{k}-$ тензор проницаемости, $\mu$ - вязкость флюида, к - коэффициент теплопроводности, $T$ - температура.

Система уравнений (1) замыкается следующим набором определяющих соотношений [1]:

$$
\begin{aligned}
& \Delta \boldsymbol{\sigma}=\mathbf{C}(1-D): \Delta \boldsymbol{\varepsilon}-\mathbf{b} \Delta p-\mathbf{C}: \alpha_{T} \Delta T-\mathbf{C}: \boldsymbol{\varepsilon}^{0}: \Delta D, \\
& \Delta m_{f}=\rho_{f} \mathbf{b}: \Delta \boldsymbol{\varepsilon}+\rho_{f} \frac{\Delta p}{M}-\alpha_{m} \rho_{f} \Delta T,
\end{aligned}
$$




$$
\begin{aligned}
& \Delta S_{s}=\mathbf{C}: \alpha_{T} \Delta \boldsymbol{\varepsilon}-\alpha_{\phi} \Delta p+\frac{C_{p s}}{T^{0}} \Delta T, \\
& \Delta \mathbf{Y}=\mathbf{C}\left(\boldsymbol{\varepsilon}^{0}+\frac{1}{2} \Delta \boldsymbol{\varepsilon}\right) \Delta \boldsymbol{\varepsilon}, \\
& \Delta \frac{1}{\rho_{f}}=\frac{1}{K_{f}} \Delta p-\alpha_{f} \Delta T, \\
& \Delta S_{f}=-\alpha_{f} \Delta p+\frac{C_{p f}}{T^{0}} \Delta T,
\end{aligned}
$$

где $\mathbf{C}$ - тензор упругих коэффициентов, $\mathbf{b}-$ коэффициент Био, $\mathbf{C}: \alpha_{T}-$ тензор термоупругих коэффициентов, $\boldsymbol{\varepsilon}=\left(\nabla \otimes \xi+(\nabla \otimes \xi)^{T}\right) / 2-$ тензор деформаций, $\xi$ - вектор перемещений скелета, $M$ - модуль Био, $\alpha_{\phi}, \alpha_{f}-$ коэффициенты термического расширения скелета и флюида соответственно, $C_{p s}$, $C_{p f}$ - теплоемкость скелета и флюида, $\mathbf{Y}$ - обобщенная термодинамическая сила, связанная с повреждаемостью, $K_{f}$ - модуль объемного сжатия флюида, $S_{\alpha}$ - энтропия фазы $\alpha, D$ - параметр повреждаемости, $\Delta f=f-f^{0}, f^{0}-$ опорное значение параметра $f$.

Выражение для внутренней энергии скелета имеет вид

$$
\Delta E_{s}=\Delta E_{s \varepsilon}+\Delta E_{s p}+\Delta E_{s T}+\Delta E_{s D},
$$

где

$$
\begin{aligned}
\Delta E_{s \varepsilon} & =\left[\boldsymbol{\sigma}^{0}+\frac{1}{2} \mathbf{C}: \Delta \boldsymbol{\varepsilon}+p^{0} \mathbf{b}+T^{0} \mathbf{C}: \alpha_{T}\right] \Delta \boldsymbol{\varepsilon}, \\
\Delta E_{s p} & =\left[\frac{1}{N}\left(p-\frac{1}{2} \Delta p\right)-\alpha_{\varphi}\left(T-\frac{1}{2} \Delta T\right)\right] \Delta p, \\
\Delta E_{s T} & =\left[-\alpha_{\varphi}\left(p-\frac{1}{2} \Delta p\right)+\frac{C_{p s}}{T^{0}}\left(T-\frac{1}{2} \Delta T\right)\right] \Delta T, \\
\Delta E_{s D} & =-\left[\mathbf{Y}^{0}+\mathbf{C}\left(\boldsymbol{\varepsilon}^{0}+\frac{1}{2} \Delta \boldsymbol{\varepsilon}\right) \Delta \boldsymbol{\varepsilon}\right] \Delta \mathbf{D} .
\end{aligned}
$$

Выражение для внутренней энергии флюида имеет вид

$$
\begin{aligned}
& \Delta E_{f}=\left[\frac{\phi}{K_{f}}\left(p-\frac{1}{2} \Delta p\right)-\phi \alpha_{f}\left(T-\frac{1}{2} \Delta T\right)\right] \Delta p- \\
& -\left[\phi \alpha_{f}\left(p-\frac{1}{2} \Delta p\right)-\frac{C_{p f}}{T^{0}}\left(T-\frac{1}{2} \Delta T\right)\right] \Delta T, \quad \text { где } \phi=m_{f} / \rho_{f}-\text { пористость. }
\end{aligned}
$$


Коэффициенты в определяющих соотношениях могут являться функциями параметров состояния среды $\chi=\{\varepsilon, p, T, D\}$. В связи с этим в модель был добавлен учет зависимости проницаемости пласта и вязкости флюида от текущих параметров состояния $\mathbf{k}=\mathbf{k}(\boldsymbol{\varepsilon}, p, T, D), \mu=\mu(p, T)$.

Первичными неизвестными системы уравнений (1) являются перемещения $\xi$, давление $p$ и температура $T$. Задача рассматривается в трехмерной области $\Omega$ с границей $\partial \Omega$, на которой для каждого параметра определены граничные условия Дирихле $\left(\partial \Omega_{D}\right)$ или Неймана $\left(\partial \Omega_{N}\right), \partial \Omega=\partial \Omega_{N} \cup \Omega_{D}$. Условие Дирихле $\left.x\right|_{\partial \Omega_{D}}=0$ - жестко закрепленная граница области, $\left.p\right|_{\partial \Omega_{D}}=\tilde{p}$ - давление на границе области, $\left.T\right|_{\partial \Omega_{D}}=\tilde{T}$ - температура на границе области. Условие Неймана $\boldsymbol{\sigma}:\left.\mathbf{n}\right|_{\partial \Omega_{N}}=\tilde{\mathbf{t}}-$ вектор нормальных напряжений на границе области, $\left.\mathbf{q} \cdot \mathbf{n}\right|_{\partial \Omega_{N}}=\tilde{q}-$ поток флюида через границу области, $\left.\mathbf{q}_{T} \cdot \mathbf{n}\right|_{\partial \Omega_{N}}=\tilde{q}_{T}$ - поток тепла через границу области.

Стоит отметить, что для каждой переменной $\xi, p, T$ области $\partial \Omega_{N}$ и $\partial \Omega_{D}$ - свои, то есть для переменной $\alpha: \partial \Omega=\partial \Omega_{N}^{(\alpha)} \cup \partial \Omega_{D}^{(\alpha)}$. Однако для упрощения дальнейших выкладок будем считать, что они совпадают.

\section{3. Слабая постановка задачи}

Пусть переменные задачи ( $\xi, p, T, D)$ являются элементами пространств $V_{\alpha}$ достаточно гладких функций, удовлетворяющих граничному условию Дирихле. Для построения слабой постановки задачи умножим каждое уравнение в системе (1) на соответствующую пробную функцию и проинтегрируем по области $\Omega$. Тогда в соответствии с формулой Грина с учетом определяющих соотношений система уравнений (1) примет вид

$$
\begin{gathered}
\int_{\Omega}(\mathbf{L v})^{T}: \mathbf{C}(1-D): \boldsymbol{\varepsilon}(\boldsymbol{\xi}) d \Omega-\int_{\Omega}(\mathbf{L v})^{T} \mathbf{b} p d \Omega-\int_{\Omega}(\mathbf{L v})^{T}: \mathbf{C}: \alpha_{T} T d \Omega=\int_{\partial \Omega_{N}} \mathbf{v}^{T} \tilde{\mathbf{t}} d S \\
\int_{\Omega} v \mathbf{b}: \mathbf{L} \frac{\partial \xi}{\partial t} d \Omega+\int_{\Omega} v \frac{1}{M} \frac{\partial p}{\partial t} d \Omega-\int_{\Omega} v \alpha_{m} \frac{\partial T}{\partial t} d \Omega+\int_{\Omega}(\operatorname{grad} v)^{T} \frac{\mathbf{k}}{\mu} \operatorname{grad}(p) d \Omega=-\int_{\partial \Omega_{N}} v \tilde{q} d \Omega \\
\int_{\Omega} v\left[\frac{\partial E_{S}}{\partial t}+\frac{\partial E_{f}}{\partial t}\right] d \Omega-\int_{\Omega}(\operatorname{grad} v)^{T} \frac{1}{\phi} E_{f} \mathbf{w} d \Omega-\int_{\Omega}(\operatorname{grad} v)^{T} p \mathbf{w} d \Omega- \\
-\int_{\Omega}(\operatorname{grad} v)^{T} \mathbf{q}_{T} d \Omega=-\int_{\partial \Omega_{N}} v \rho_{f} e_{f} \tilde{q} d S-\int_{\partial \Omega_{N}} v p \tilde{q} d S-\int_{\partial \Omega_{N}} v \tilde{q}_{T} d S
\end{gathered}
$$

где $\mathbf{v} \in V_{\alpha}^{0}$ - пробные функции, определенные в области $\Omega$, такие что $\left.\mathbf{v}\right|_{\partial \Omega_{D}}=0$. 
Симметричные тензоры деформаций и напряжений представим с помощью векторных обозначений Фойгта [21]:

$$
\varepsilon=\left[\varepsilon_{x}, \varepsilon_{y}, \varepsilon_{z}, \gamma_{x y}, \gamma_{y z}, \gamma_{x z}\right]^{T}, \quad \gamma_{i j}=2 \varepsilon_{i j}, \quad \sigma=\left[\sigma_{x}, \sigma_{y}, \sigma_{z}, \sigma_{x y}, \sigma_{y z}, \sigma_{x z}\right]^{T} .
$$

В этом случае связь между напряжением и деформацией для изотропной среды может быть записана в виде $\boldsymbol{\sigma}=\mathbf{C}: \boldsymbol{\varepsilon}, \boldsymbol{\varepsilon}=\mathbf{L} \boldsymbol{\xi}$, где $\boldsymbol{\xi}=\left[\xi_{x}, \xi_{y}, \xi_{z}\right]^{T}$,

$$
\mathbf{C}=\frac{E}{(1+v)(1-2 v)}\left[\begin{array}{cccccc}
1-v & v & v & 0 & 0 & 0 \\
v & 1-v & v & 0 & 0 & 0 \\
v & v & 1-v & 0 & 0 & 0 \\
0 & 0 & 0 & (1-2 v) / 2 & 0 & 0 \\
0 & 0 & 0 & 0 & (1-2 v) / 2 & 0 \\
0 & 0 & 0 & 0 & 0 & (1-2 v) / 2
\end{array}\right],
$$$$
\mathbf{L}=\left[\begin{array}{cccccc}
\frac{\partial}{\partial x} & 0 & 0 & \frac{\partial}{\partial y} & 0 & \frac{\partial}{\partial z} \\
0 & \frac{\partial}{\partial y} & 0 & \frac{\partial}{\partial x} & \frac{\partial}{\partial z} & 0 \\
0 & 0 & \frac{\partial}{\partial z} & 0 & \frac{\partial}{\partial y} & \frac{\partial}{\partial x}
\end{array}\right]^{T}
$$

Воспользовавшись выражениями (3) для внутренней энергии скелета, найдем её производную по времени:

$$
\begin{aligned}
& \frac{\partial E_{S}}{\partial t}=\left[\mathbf{C}(1-\Delta D): \Delta \boldsymbol{\varepsilon}+p^{0} \mathbf{b}+T^{0} \mathbf{C}: \alpha_{T}\right] \frac{\partial \boldsymbol{\varepsilon}}{\partial t}+ \\
& +\left[\frac{1}{N} p-\alpha_{\varphi} T\right] \frac{\partial p}{\partial t}+\left[-\alpha_{\varphi} p+\frac{C_{p s}}{T^{0}} T\right] \frac{\partial T}{\partial t}-\left[\frac{1}{2} \frac{d D}{d t} \mathbf{C}: \boldsymbol{\varepsilon}\right] \boldsymbol{\varepsilon} .
\end{aligned}
$$

В последней формуле использовано допущение, что $\boldsymbol{\varepsilon}^{0}=0, D^{0}=0$ и $\mathbf{Y}^{0}=0$. Аналогично для внутренней энергии флюида (4) получим

$$
\frac{\partial E_{f}}{\partial t}=\left[\frac{\phi}{K_{f}} p-\phi \alpha_{f} T\right] \frac{\partial p}{\partial t}+\left[-\phi \alpha_{f} p+\frac{C_{p f}}{T^{0}} T\right] \frac{\partial T}{\partial t} .
$$

Таким образом, производная полной внутренней энергии по времени записывается как $\partial E / \partial t=\partial E_{s} / \partial t+\partial E_{f} / \partial t$, где вид слагаемых определен выше. 


\section{4. Конечномерные аппроксимации}

Рассмотрим пространственную аппроксимацию системы уравнений (5). Введем конечномерные пространства $V_{\alpha}^{h} \subset V_{\alpha}, V_{\alpha}^{0, h} \subset V_{\alpha}^{0}$, причем $V_{\alpha}^{h}=$ $=\operatorname{span}\left(\phi_{i}^{(\alpha)}\right)$, где $\phi_{i}^{(\alpha)}-$ базисные функции. Тогда для произвольной функции $f$ имеем

$$
f=\sum_{i=1}^{N_{\alpha}} \phi_{i}^{(\alpha)} f_{i}, \quad f=\xi, p, T .
$$

Соответственно, аппроксимация системы (5) в матричном виде записывается как

$\left[\begin{array}{ccc}-\mathbf{A}_{\xi \xi} & \mathbf{A}_{\xi p} & -\mathbf{A}_{\xi T} \\ \mathbf{A}_{\xi p}^{T} & \mathbf{A}_{p p} & \mathbf{A}_{p T} \\ \mathbf{A}_{T \xi} & \mathbf{A}_{T p} & \mathbf{A}_{T T}\end{array}\right]\left[\begin{array}{c}\partial \xi / \partial t \\ \partial p / \partial t \\ \partial T / \partial t\end{array}\right]+\left[\begin{array}{ccc}0 & 0 & 0 \\ 0 & \mathbf{B}_{p p} & 0 \\ \mathbf{B}_{T \xi} & \mathbf{B}_{T p} & \mathbf{B}_{T T}\end{array}\right]\left[\begin{array}{c}\xi \\ p \\ T\end{array}\right]=\left[\begin{array}{c}-\partial \mathbf{f}_{\xi} / \partial t \\ \mathbf{f}_{p} \\ \mathbf{f}_{T}\end{array}\right]$,

где используются следующие обозначения:

$$
\begin{aligned}
& {\left[\mathbf{A}_{\xi \xi}\right]_{i j}=\int_{\Omega}\left[\mathbf{L} \phi_{\xi}\right]_{i k}[\mathbf{C}(1-D)]_{k l}\left[\mathbf{L} \phi_{\xi}\right]_{l j} d \Omega,} \\
& {\left[\mathbf{A}_{\xi p}\right]_{i j}=\left[\mathbf{A}_{p \xi}\right]_{j i}=\int_{\Omega}\left[\mathbf{L} \phi_{\xi}\right]_{i k} \mathbf{b}_{k} \phi_{p, j} d \Omega,} \\
& {\left[\mathbf{A}_{\xi T}\right]_{i j}=-\int_{\Omega}\left[\mathbf{L} \phi_{\xi}\right]_{i k}\left[\mathbf{C}(1-D): \alpha_{T}\right]_{k l} \phi_{T, j} d \Omega,} \\
& {\left[\mathbf{B}_{p p}\right]_{i j}=\int_{\Omega} \nabla \phi_{p, i} \frac{\mathbf{k}}{\mu} \nabla \phi_{p, j} d \Omega, \quad\left[\mathbf{A}_{p p}\right]_{i j}=\int_{\Omega} \phi_{p, i} \frac{1}{M} \phi_{p, j} d \Omega,} \\
& {\left[\mathbf{A}_{p T}\right]_{i j}=-\int_{\Omega} \phi_{p, i} \alpha_{m} \phi_{T, j} d \Omega,} \\
& {\left[\mathbf{A}_{T \xi}\right]_{i j}=\int_{\Omega} \phi_{T, i}\left[\sigma^{0}+\mathbf{C}(1-D): \boldsymbol{\varepsilon}+p^{0} \mathbf{b}+T^{0} \mathbf{C}: \alpha_{T}\right]_{i k}\left[\mathbf{L} \phi_{\xi}\right]_{k j} d \Omega,} \\
& {\left[\mathbf{A}_{T p}\right]_{i j}=\int_{\Omega} \phi_{T, i}\left(\frac{1}{M} p-\alpha{ }_{m} T\right) \phi_{p, j} d \Omega,} \\
& {\left[\mathbf{A}_{T T}\right]_{i j}=\int_{\Omega} \phi_{T, i}\left[-\alpha_{m} p+\left(C{ }_{p s}+C_{p f}\right) \frac{T}{T}\right]_{i j} \phi_{T, j} d \Omega,} \\
& {\left[\mathbf{B}_{T \xi}\right]_{i j}=\int_{\Omega} \nabla \phi_{T, i}\left[\frac{1}{2} \frac{d D}{d t} \mathbf{C}: \boldsymbol{\varepsilon}\right]_{i k}\left[\mathbf{L} \phi_{\xi}\right]_{k j} d \Omega,} \\
& {\left[\mathbf{B}_{T p}\right]_{i j}=\int_{\Omega} \nabla \phi_{T, i}\left\{\frac{E_{f}^{0}}{\varphi}+\left[\frac{1}{K_{f}} p-\alpha_{f} T\right]_{\Delta p-\left[\alpha_{f} p-\frac{C_{p f}}{f}\right] \Delta T}\right\}_{i j} \frac{\mathbf{k}}{\mu} \nabla \phi_{p, j} d \Omega,}
\end{aligned}
$$




$$
\begin{aligned}
& {\left[\mathbf{B}_{T T}\right]_{i j}=\int_{\Omega} \nabla \phi_{T, i} \mathbf{\kappa} \nabla \phi_{T, j} d \Omega, \quad \mathbf{f}_{\xi, i}=\int_{\partial \Omega_{N}}\left[\phi_{\xi}\right]_{i j} \tilde{\mathbf{t}}_{j} d S, \quad \mathbf{f}_{p, i}=-\int_{\partial \Omega_{N}} \phi_{p, i} \tilde{q} d \Omega,} \\
& \mathbf{f}_{T, i}=-\int_{\partial \Omega_{N}} \phi_{T, i} \rho_{f} e_{f} \tilde{q} d S-\int_{\partial \Omega_{N}} \phi_{T, i} p \tilde{q} d S-\int_{\partial \Omega_{N}} \phi_{T, i} \tilde{q}_{T} d S .
\end{aligned}
$$

Вычисление интегралов в уравнении (7) производится с использованием квадратурных формул Гаусса второго порядка [22].

Для дискретизации уравнения (8) по пространству использовалась тетраэдральная сетка с квадратичными базисными функциями для перемещений и линейными для давления и температуры (элементы Тейлора-Худа [14]).

Для аппроксимации системы уравнений по времени будем использовать полностью неявную относительно перемещений $x$, давления $p$ и температуры $T$ схему. Параметр повреждаемости $D$ будет учитываться явным образом. Пусть $f=f(t)$ - значение какой-либо величины в момент времени $t$, тогда $\hat{f}=f(t+\Delta t)$ - её значение в момент времени $t+\Delta t$. Соответственно, система уравнений (6) после аппроксимации по времени примет вид

$$
\begin{aligned}
& {\left[\begin{array}{ccc}
-\mathbf{A}_{\xi \xi} & \mathbf{A}_{\xi p} & -\mathbf{A}_{\xi T} \\
\mathbf{A}_{\xi p}^{T} & \mathbf{A}_{p p}+\Delta t \mathbf{B}_{p p} & \mathbf{A}_{p T} \\
\mathbf{A}_{T \xi}+\Delta t \mathbf{B}_{T \xi} & \mathbf{A}_{T p}+\Delta t \mathbf{B}_{T p} & \mathbf{A}_{T T}+\Delta t \mathbf{B}_{T T}
\end{array}\right] \cdot\left[\begin{array}{l}
\hat{\xi} \\
\hat{p} \\
\hat{T}
\end{array}\right]=} \\
& =\left[\begin{array}{ccc}
-\mathbf{A}_{\xi \xi} & \mathbf{A}_{\xi p} & -\mathbf{A}_{\xi T} \\
\mathbf{A}_{\xi p}^{T} & \mathbf{A}_{p p} & \mathbf{A}_{p t} \\
\mathbf{A}_{T \xi} & \mathbf{A}_{T p} & \mathbf{A}_{T T}
\end{array}\right] \cdot\left[\begin{array}{c}
\xi \\
p \\
T
\end{array}\right]+\Delta t\left[\begin{array}{c}
-\Delta \mathbf{f}_{\xi} / \Delta t \\
\mathbf{f}_{p} \\
\mathbf{f}_{T}
\end{array}\right] .
\end{aligned}
$$

В связи с тем что система уравнений (8) является нелинейной, для её решения используется метод Ньютона [23]. Критерием сходимости итерационного алгоритма является одновременное выполнение следующих условий:

$$
\|\Delta \mathbf{x}\|_{\infty}<\varepsilon_{x}, \quad\|\mathbf{R}\|_{\infty}<\varepsilon_{R},
$$

где $\Delta \mathbf{x}$ - величина приращения неизвестных на текущей итерации, $\mathbf{R}-$ значение нелинейной невязки, $\varepsilon_{x}, \varepsilon_{R}-$ параметры.

С учетом вида матриц (7) якобиан системы (8) имеет вид:

$$
\mathbf{J}=\left[\begin{array}{ccc}
-\mathbf{A}_{\xi \xi} & \mathbf{A}_{\xi p} & -\mathbf{A}_{\xi T} \\
\mathbf{A}_{\xi p}^{T} & \mathbf{A}_{p p}+\Delta t \mathbf{B}_{p p} & \mathbf{A}_{p T} \\
\mathbf{J}_{T \xi} & \mathbf{J}_{T p} & \mathbf{J}_{T T}
\end{array}\right],
$$




$$
\begin{aligned}
& \mathbf{J}_{T \xi}=\mathbf{A}_{T \xi}+\Delta t \mathbf{B}_{T \xi}+\frac{\partial \mathbf{A}_{T \xi}}{\partial \xi} \Delta \xi+\Delta t \mathbf{B}_{T \xi} \hat{\xi}, \\
& \mathbf{J}_{T p}=\mathbf{A}_{T p}+\Delta t \mathbf{B}_{T p}+\frac{\partial \mathbf{A}_{T p}}{\partial p} \Delta p+\frac{\partial \mathbf{A}_{T T}}{\partial p} \Delta T+\Delta t \frac{\partial \mathbf{B}_{T p}}{\partial p} \hat{p}, \\
& \mathbf{J}_{T T}=\mathbf{A}_{T T}+\Delta t \mathbf{B}_{T T}+\frac{\partial \mathbf{A}_{T p}}{\partial T} \Delta p+\frac{\partial \mathbf{A}_{T T}}{\partial T} \Delta T+\Delta t \frac{\partial \mathbf{B}_{T p}}{\partial T} \hat{p}
\end{aligned}
$$

И

$$
\begin{aligned}
& \frac{\partial \mathbf{A}_{T \xi}}{\partial \xi}=\int_{\Omega} \phi_{T}^{T}[\mathbf{C}(1-D): \mathbf{I}]\left(\mathbf{L} \phi_{\xi}\right) d \Omega, \quad \frac{\partial \mathbf{A}_{T p}}{\partial p}=\int_{\Omega} \phi_{T}^{T} \frac{1}{M} \phi_{p} d \Omega, \\
& \frac{\partial \mathbf{A}_{T p}}{\partial T}=-\int_{\Omega} \phi_{T}^{T} \alpha_{m} \phi_{p} d \Omega, \quad \frac{\partial \mathbf{A}_{T T}}{\partial p}=-\int_{\Omega} \phi_{T}^{T} \alpha_{m} \phi_{T} d \Omega, \\
& \frac{\partial \mathbf{A}_{T T}}{\partial T}=\int_{\Omega} \phi_{T}^{T} \frac{C_{p s}+C_{p f}}{T^{0}} \phi_{T} d \Omega, \quad \frac{\partial \mathbf{B}_{T \xi}}{\partial \xi}=\int_{\Omega} \nabla \phi_{T}^{T}\left[\frac{\partial}{\partial \xi}\left(\frac{1}{2} \frac{d D}{d t} \mathbf{C}: \boldsymbol{\varepsilon}\right)\right]\left(\mathbf{L} \phi_{\xi}\right) d \Omega, \\
& \frac{\partial \mathbf{B}_{T p}}{\partial p}=\int_{\Omega}\left(\mathbf{L} \phi_{\xi}\right)^{T}\left[\frac{1}{K_{f}}\left(2 p-p^{0}\right)-\alpha_{f}\left(2 T-T^{0}\right)\right] \frac{\mathbf{k}}{\mu} \nabla \phi_{p} d \Omega, \\
& \frac{\partial \mathbf{B}_{T p}}{\partial T}=\int_{\Omega}\left(\mathbf{L} \phi_{\xi}\right)^{T}\left[\frac{C_{p f}}{\phi}-\alpha_{f}\left(2 p-p^{0}\right)\right] \frac{\mathbf{k}}{\mu} \nabla \phi_{p} d \Omega .
\end{aligned}
$$

Решение системы линейных уравнений (8) на каждой ньютоновской итерации производится с использованием стабилизированного метода бисопряженных градиентов (BiCGStab) [24]. В качестве предобуславливателя использовалось неполное LU разложение с одноуровневым заполнением (ILU(1)).

Для обеспечения устойчивости конечномерной задачи применялся ряд подходов, в соответствии с которыми преобразовывалась матрица системы (8). Для матриц масс $\mathbf{A}_{p p}, \mathbf{A}_{p T}, \mathbf{A}_{T p}$ и $\mathbf{A}_{T T}$ использовался метод диагонализации матриц масс (mass lumping, [25]). Для уменьшения ширины ленты разреженной матрицы системы использовался алгоритм Катхилла-Макки [26].

\section{5. Результаты моделирования}

В данном разделе приведены результаты моделирования с использованием программного комплекса, в основе которого лежит математическая модель и вычислительный алгоритм, описанные ранее. Валидация алгоритма проводилась на ряде тестов (задача Терцаги, тест Манделя и тест на одномерное неизотермическое расширение), для которых известно аналитическое решение [27]. Ниже приведены результаты моделирования ряда задач в реалистичных постановках. 
При проведении расчетов использовались следующие настройки сходимости ньютоновских итераций: $\varepsilon_{x}=0.001, \varepsilon_{R}=0.001$. В случае если количество итераций на одном временном шаге превышает 5 , временной шаг уменьшается в 2 раза и итерационный алгоритм повторяется с начала. В случае успешной сходимости алгоритма осуществляется расчет следующего временного периода с постепенным увеличением шага по времени. Критерием остановки линейного итерационного процесса является достижение нормы невязки величины $10^{-5}$.

Для моделирования зависимости проницаемости от параметров состояния среды используется зависимость [28]:

$$
k=k_{0} \exp [-\beta(\tilde{\sigma}-\alpha p)],
$$

где $k_{0}$ - начальное значение проницаемости, $\tilde{\sigma}=1 / 3\left(\sigma_{x}+\sigma_{y}+\sigma_{z}\right)-$ среднее напряжение. В качестве значений коэффициентов $\alpha$ и $\beta$ для дальнейших расчетов были приняты значения из [28]: $\alpha=0.1 \Pi^{-1}, \beta=0.05 \Pi^{-1}$.

Для описания зависимости вязкости от температуры используется корреляция Беггса-Робинсона [29]:

$$
\mu=10^{X}-1, \quad X=10^{Z} T^{-1.163}, \quad Z=3.0324-0.02023 \gamma_{0},
$$

где $\mu$ - вязкость порового флюида в сП (1 сП =0.001 Па·с $), \gamma_{0}-$ плотность флюида в ${ }^{\circ} A P I$.

Для описания эволюции параметра повреждаемости используется явная зависимость [30]:

$$
D= \begin{cases}0, & \tilde{\varepsilon}<\tilde{\varepsilon}_{c}, \\ \frac{D_{\text {off }}}{\tilde{\varepsilon}_{\text {off }}-\tilde{\varepsilon}_{c}} \tilde{\varepsilon}-D_{\text {off }} \frac{\varepsilon_{c}}{\tilde{\varepsilon}_{\text {off }}-\tilde{\varepsilon}_{c}}, & \tilde{\varepsilon}_{c} \leqslant \tilde{\varepsilon} \leqslant \tilde{\varepsilon}_{\text {off }}, \\ D_{\text {lim }}-\left(D_{\text {lim }}-D_{\text {off }}\right) \frac{\tilde{\varepsilon}_{\text {off }}}{\tilde{\varepsilon}}, & \tilde{\varepsilon}>\tilde{\varepsilon}_{\text {off }},\end{cases}
$$

где $\varepsilon_{i}$ - главные деформации, $\tilde{\varepsilon}=\sqrt{\sum_{i=1}^{3}\left\langle\varepsilon_{i}\right\rangle^{2}},\left\langle\varepsilon_{i}\right\rangle=\left(\varepsilon_{i}+\left|\varepsilon_{i}\right|\right) / 2$. Параметры зависимости (11) принимались равными: $\tilde{\varepsilon}_{c}=0.0002, \quad \tilde{\varepsilon}_{\text {off }}=0.003, D_{\text {lim }}=1$, $D_{\text {off }}=0.9$.

Значения прочих входных параметров для всех представленных моделей приведены в табл.1. 
Таблица 1. Значения входных параметров для модели термического воздействия на пласт.

\begin{tabular}{|c|c|}
\hline Параметр & Значение \\
\hline Модуль Юнга, $E$ & 20 ГПа \\
\hline Коэффициент Пуассона, $v$ & 0.3 \\
\hline Модуль Био, $N$ & 10 ГПа \\
\hline Модуль объемного сжатия флюида, $K_{f}$ & 3.3 ГПа \\
\hline Коэффициент Био, $b$ & 0.79 \\
\hline Проницаемость, $k$ & $1 \cdot 10^{-16} \mathrm{~m}^{2}$ \\
\hline Пористость, $\varphi$ & 0.1 \\
\hline Вязкость, $\mu$ & $1 \mathrm{мПа} \cdot \mathrm{c}$ \\
\hline Плотность скелета, $\rho_{s}$ & 2100 кг/м \\
\hline Плотность флюида, $\rho_{f}$ & 1000 кг/м \\
\hline Коэффициент объемного температурного расширения скелета, $\alpha_{s}$ & $1 \cdot 10^{-6} 1 / \mathrm{K}$ \\
\hline Коэффициент объемного температурного расширения флюида, $\alpha_{f}$ & $1 \cdot 10^{-4} 1 / \mathrm{K}$ \\
\hline Удельная теплоемкость скелета, $c_{p s}$ & 1000 Дж/(кГ $\cdot \mathrm{K})$ \\
\hline Удельная теплоемкость флюида, $c_{p f}$ & 4200 Дж/(кГ $\cdot \mathrm{K})$ \\
\hline Эффективная теплопроводность, $\kappa$ & $2 \mathrm{Bт} \mathrm{/} \mathrm{(м} \cdot \mathrm{K})$ \\
\hline
\end{tabular}

5.1. Моделирование термического воздействия на пласт с добывающей и нагнетательной скважиной. В данном расчете моделируется реакция пласта при использовании системы поддержания пластового давления, при котором нагнетательная скважина закачивает флюид при температуре $400^{\circ} \mathrm{C}$ с приемистостью $0.2 \mathrm{~m}^{3} /$ сут. Добывающая скважина работает с постоянным забойным давлением 100 бар. Начальное пластовое давление 200 бар, начальная температура $-100^{\circ} \mathrm{C}$, напряжения по осям $x, y$ и $z-$ 300, 330 и 550 бар соответственно, начальные деформации равны нулю.

Рассматривается изотропный пласт размером $100 \times 100 \times 1$ м. Скважины расположены на противоположных концах диагонали пласта. Для более корректного учета эффектов, возникающих в прискважинной зоне, расчетная сетка имеет локальное измельчение вдоль границы модели. Механические граничные условия: $\xi_{x}(x=0)=0, \xi_{y}(y=0)=0, \xi_{z}(z=0)=0, \mathbf{t}_{x}(x=100)=300$ бар, $\mathbf{t}_{y}(y=100)=330$ бар, $\mathbf{t}_{z}(z=1)=550$ бар. Фильтрационные и термические граничные условия: термически изолированная непроницаемая граница.

Моделировалась разработка пласта в течении 5 лет. По результатам расчетов оценивалось изменение давления, температуры, параметра повреждаемости и проницаемости со временем. Распределения полей соот- 
ветствующих величин в латеральном разрезе через 6,12 и 60 месяцев приведены на рис.1. Распределение величин в вертикальном срезе не приводится ввиду постоянства свойств в вертикальном направлении.

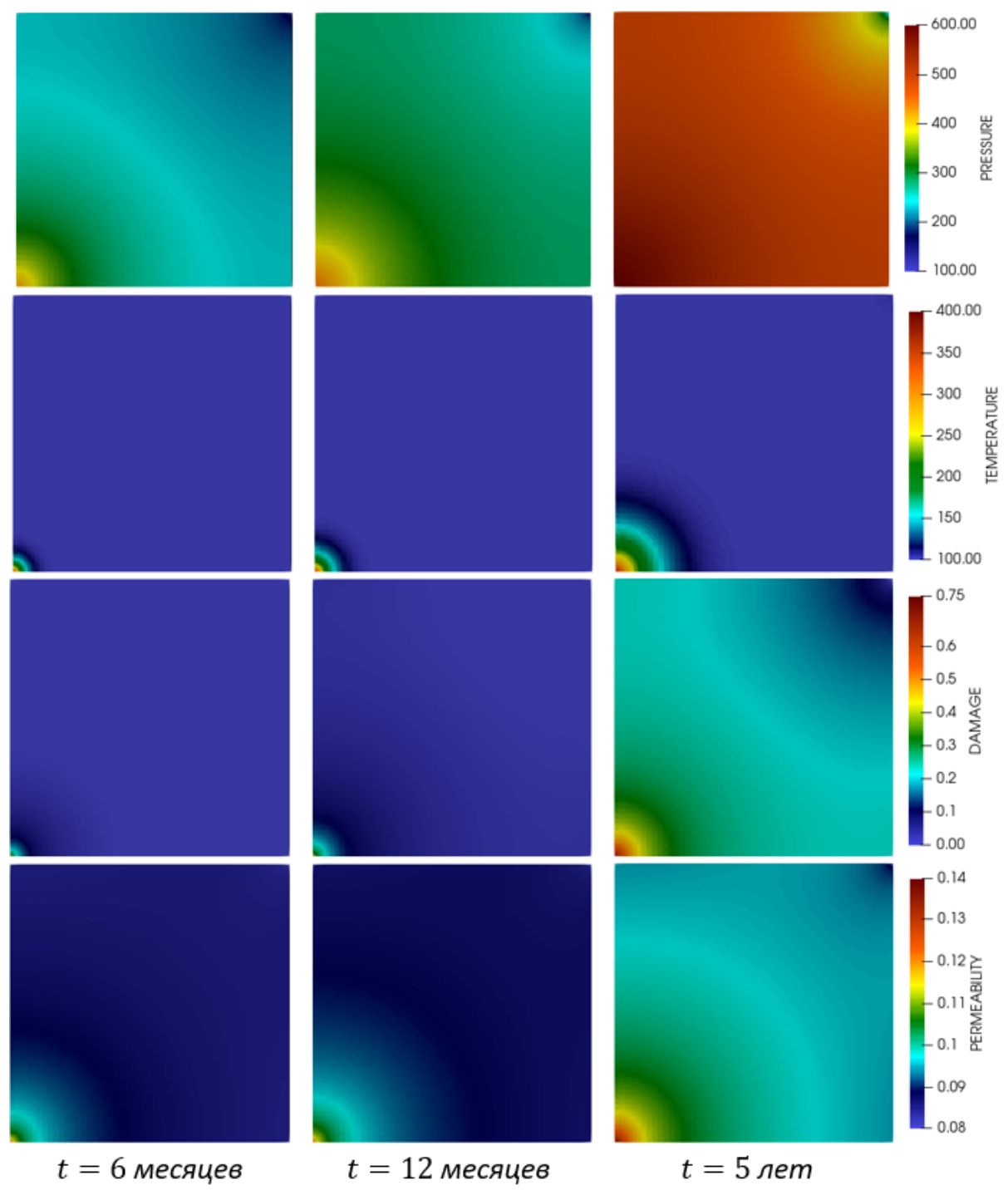

Рис.1. Распределение давления $p$ (1 ряд), температуры $T$ (2 ряд), параметра повреждаемости $D$ (3 ряд), проницаемости $k$ (4 ряд) через 6 месяцев (слева), 12 месяцев (по центру) и 5 лет (справа).

Также был проведен расчет данной модели при отсутствии учета повреждаемости. Сравнение распределений компоненты $\varepsilon_{x x}$ тензора деформаций на момент времени 5 лет для случаев учета и в отсутствие учета повреждаемости приведено на рис.2. Из данного рисунка видно, что учет раз- 
рушения породы существенно влияет на расчет напряженно-деформированного состояния (для данного теста различие в значениях тензора деформаций достигает $20 \%)$.
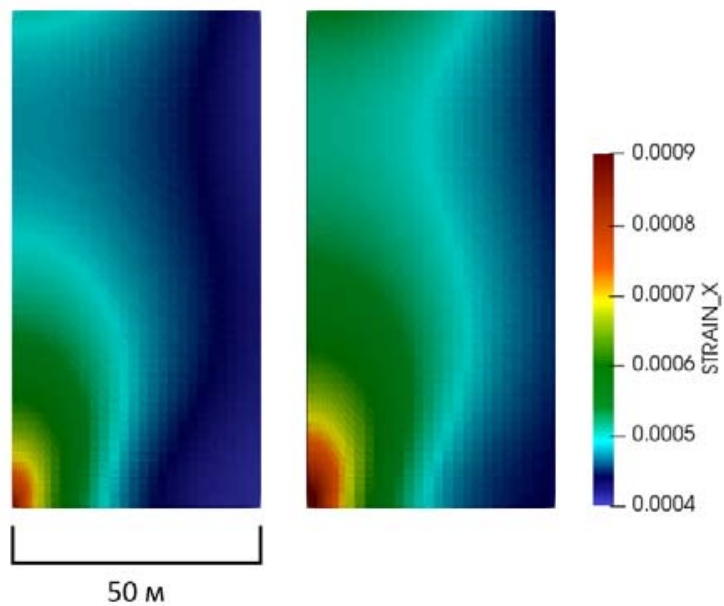

Рис.2. Компонента $\varepsilon_{x x}$ тензора деформаций на момент времени 5 лет при учете (слева) и в отсутствие учета (справа) повреждаемости.

\section{2. Моделирование развития повреждаемости вблизи нагнетатель-} ной скважины. В следующем расчете рассматривается разрушение призабойной зоны нагнетательной скважины при закачке теплоносителя в пласт при высоком давлении в начальные моменты времени. Для оценки возникающих эффектов был проведен численный расчет на модели размером $0.5 \times 0.5 \times 0.2 \mathrm{M}$, в углу которой расположена скважина радиусом $0.1 \mathrm{M}$, нагнетающая флюид при температуре $300^{\circ} \mathrm{C}$ с постоянной приемистостью $1 \mathrm{~m}^{3} /$ сут. Начальные и граничные условия аналогичны предыдущему случаю. Проницаемость и модуль Юнга заданы в модели в соответствии с распределением Гаусса. Расчетная сетка построена с учетом геометрии скважины, общее количество конечных элементов сетки составляет 59508, узлов - 120183.

На рис. 3 приведены поля динамических параметров на момент времени 12 часов. По результатам расчета можно видеть практически полное разрушение коллектора в радиусе $0.2 \mathrm{M}$ от скважины. При этом разрушение в большей степени вызвано повышенным пластовым давлением (более 800 бар), поскольку увеличение температуры в данной зоне незначительно. Также получено увеличение проницаемости в 2 раза по сравнению с начальной. 

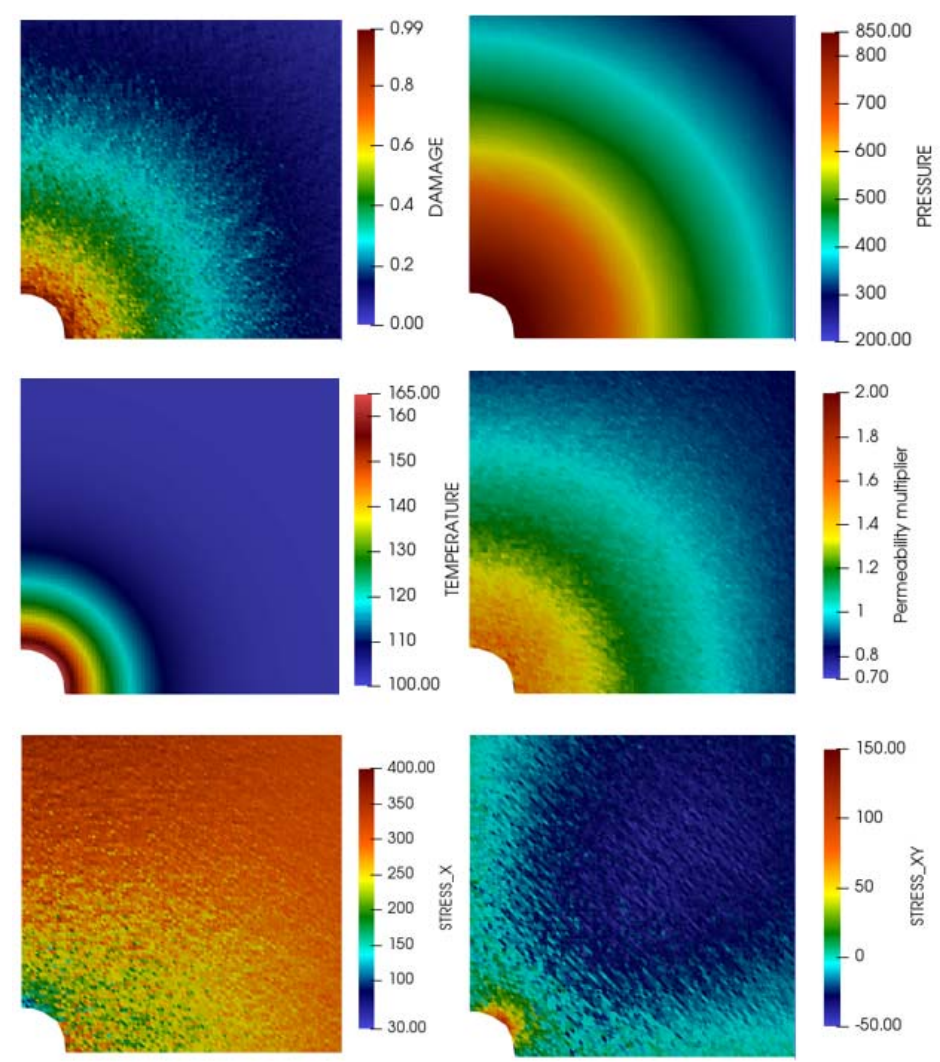

Рис.3. Распределение параметра повреждаемости $D$, давления $p$, температуры $T$, отношения текущей и начальной проницаемости $k / k^{0}$, а также латеральных компонентов тензора напряжений $\sigma_{x x}$ и $\sigma_{x y}$ через 12 часов.

Для данной модели была проведена оценка влияния проницаемости на распределение поля давления. На рис.4 приведено сравнение распределения поля давления в случае учета изменения проницаемости от состояния среды (слева), а также в случае неизменной проницаемости (справа). В результате проведенных расчетов получено различие в давлении более 50 бар.

\section{6. Заключение}

В настоящей работе приведена математическая модель, а также представлен вычислительный алгоритм для моделирования эволюции термопороупругой среды с учетом разрушения. Модель является обобщением модели Био и учитывает деформационные, фильтрационные и неизотермические эффекты. Разрушение среды моделируется в рамках теории континуального разрушения. Система уравнений состоит из фундаментальных законов сохранения и замыкается термодинамически согласованными определяющими соотношениями. 

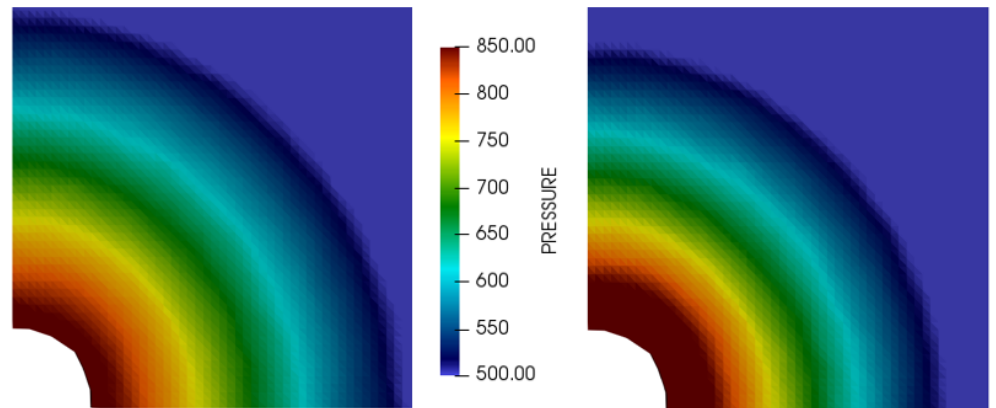

Рис.4. Сравнение поля давления на момент времени 12 часов при учете изменения проницаемости (слева) и при постоянной проницаемости (справа).

Система уравнений решается в полностью связанной постановке, вычислительный алгоритм основан на методе конечных элементов. Для аппроксимации по времени используется полностью неявная схема, в качестве конечных элементов используются тетраэдральные элементы Тэйлора-Худа.

Работа алгоритма показана на примере задач моделирования термического воздействия на нефтяной пласт. По результатам проведенных расчетов оценено влияние разрушения при различных параметрах скважин и пласта. Получено, что разрушение заметно влияет на величину упругих модулей и, как следствие, на напряженно-деформированное состояния среды.

\section{СПИСОК ЛИТЕРАТУРЫ}

1. А.С. Меретин, Е.Б. Савенков. Математическая модель фильтрационных процессов в термопороупругой среде с учетом континуального разрушения // Препринты ИПМ им. М.В. Келдыша, 2019, № 58, 38 с.

A.S. Meretin, E.B. Savenkov. Matematicheskaya model filtratsionnikh processov v termoporouprugoi srede $\mathrm{s}$ uchetom kontinualnogo razrusheniya // Preprinty IPM im. M.V. Keldysha, 2019, №58, 38 s.

2. H.F. Wang. Theory of linear poroelasticity with applications to geomechanics and hydrogeology. - Princeton: Princeton University Press, 2000.

3. S.C. Cowin. Bone poroelasticity // J. of Biomechanics, 1999, v.32, Issue 3, p.217-238.

4. A. Malandrino, E. Moeendarbary. Poroelasticity of Living Tissues // Encyclopedia of Biomedical Engineering. - Amsterdam: Elsevier, 2017, p.238-245.

5. V. Dorovsky, Yu. Perepechko. Theory of partial melting // Russian Geology and Geophysics, 1989, v.30, p.56-65.

6. V. Dorovsky, Yu. Perepechko, A. Fedorov. The Stonely Waves in the Biot-Johnson Theory and Continuum Filtration Theory // Russian Geology and Geophysics, 2012, v.53, p.621-630.

7. В.И. Голубев, А.В. Шевченко, И.Б. Петров. Об учёте водонасыщенности донных осадков в задаче морской сейсмической разведки // ДАН, 2019, т.488, №3, с.248-252; V.I. Golubev, A.V. Shevchenko, I.B. Petrov. Taking into Account Fluid Saturation of Bottom Sediments in Marine Seismic Survey // DAN, 2019, v.488, № 3, p.248-252.

8. L.W. Lake. Enhanced oil recovery. - New York: Prentice Hall, 1989.

9. Ю.Н. Работнов. Механизм длительного разрушения // Вопросы прочности материалов и конструкций. - М.: АН СССР, 1959, с.5-7; 
Yu.N. Rabotnov. Mehanizm dlitelnogo razrusheniya // Voposy prochnosti materialov i konstruktsii. - M.:AN SSSR, 1959, s.5-7.

10. Л.М. Качанов. О времени разрушения в условиях ползучести // Изв. АН СССР. ОТН., 1958, № 8, c.26-31;

L.M. Kachanov. O vremeni razrusheniya v usloviiach polzuchesti // Izv. AN SSSR. OTN., 1958, №8, s.26-31.

11. J. Kim, H.A. Tchelepi, R. Juanes. Stability, Accuracy and Efficiency of Sequential Methods for Coupled Flow and Geomechanics // SPE Paper 119084, 2009.

12. F. Brezzi, M. Fortin. Mixed and Hybrid Finite Elements Methods. - Springer, 1991.

13. P.J. Phillips, M.F. Wheeler. Overcoming the problem of locking in linear elasticity and poroelasticity: an heuristic approach // Comp. Geosci., 2009, v.13, p.5-12.

14. C. Taylor, P.A. Hood. A numerical solution of the Navier-Stokes equations using the finite element technique // Computers and Fluids, 1973, т.1, № 1, c.73-100.

15. R.E. Showalter. Diffusion in Poro-Elastic Media // J. of Math. Analysis and Applications, 2000, v.251, № 1, p.310-340.

16. J.A. White, R.I. Borja. Stabilized low-order finite elements for coupled solid deformation/ fluid-diffusion and their application to fault zone transients // Computer Methods in Applied Mechanics and Engineering, 2008, v. 97, №49-50, p.4353-4366.

17. J. Wan. Stabilized Finite Element Method for Coupled Geomechanics and Multiphase Flow. - Stanford: PhD Thesis, Stanford Univeristy, 2002, 180 p.

18. K. Xia, A. Masud. Stabilized Finite Elements For Computational Geomechanics // ARMA/ USRMS Paper No 05-874, 2005.

19. J. Kim. Sequential methods for coupled geomechanics and multiphase flow. - Stanford University, 2010, $248 \mathrm{p}$.

20. S.E. Minkoff, N.M. Kridler. A comparison of adaptive time stepping methods for coupled flow and deformation modeling // Applied math. modelling, 2006, v.30, №9, p.993-1009.

21. W. Voigt. Lehrbuch der kristallphysik. - Leipzig: Teubner, 1928, v.962.

22. O. Zenkevich. Finite-element method in engineering. - M.: Mir., 1975, v.5.

23. M. Abramowitz, I.A. Stegun. Handbook of Mathematical Functions with Formulas, Graphs, and Mathematical Tables, 9th printing. - New York: Dover, 1972, p.18.

24. Y. Saad. Iterative methods for sparse linear systems. - Siam, 2003, v.82.

25. S.P. Neuman. Saturated-unsaturated seepage by finite elements // J. HYDRAUL. DIV., PROC., ASCE, 1973.

26. E. Cuthill, J. McKee. Reducing the bandwidth of sparse symmetric matrices // In Proc. 24th Nat. Conf. ACM, 1969, p.157-172.

27. А.С. Меретин, Е.Б. Савенков. Вычислительный алгоритм для описания эволюции термопороупругой среды с учетом разрушения // Препр. ИПМ им. М.В. Келдыша, 2019, № 82, 36 с. A.S. Meretin, E.B. Savenkov. Vychislitelnii algoritm dlya opisaniya evolutsii sredy s uchetom razrusheniya // Preprinty IPM im. M.V. Keldysha, 2019, №82, $36 \mathrm{~s}$.

28. C.A. Tang. Coupled analysis of flow, stress and damage (FSD) in rock failure // International J. of Rock Mechanics and Mining Sciences, 2002, v.39, № 4, p.477-489.

29. H.D. Beggs. Estimating the viscosity of crude oil systems // Journal of Petroleum technology, 1975, v.27, № 09, p.1140-1141.

30. J. Pogacnik, M.O'Sullivan, J.O'Sullivan. A Damage Mechanics Approach to Modeling Permeability Enhancement in Thermo-Hydro-Mechanical Simulations // Proc. of 39th Geothermal Reservoir Engineering Workshop, 2014, p. 24-26.

Поступила в редакцию 20.11.2019

После доработки 20.01.2020

Принята к публикации 27.01.2020 